\title{
Do specialist pulmonologists appropriately utilise thoracic surgery for drug-resistant pulmonary tuberculosis? A survey
}

\author{
G Alexander, ${ }^{1}$ MB ChB, FC Cardio (SA), MMed; R Perumal, ${ }^{2}$ MB ChB, MPH, MMed, FCP \\ ${ }^{1}$ Department of Cardiothoracic Surgery, Inkosi Albert Luthuli Hospital and King Dinuzulu Hospital, Durban, South Africa \\ ${ }^{2}$ Division of Pulmonology, Department of Medicine, Faculty of Health Sciences, University of Cape Town and Groote Schuur Hospital, Centre for the AIDS \\ Programme of Research in South Africa, Cape Town, South Africa
}

Corresponding author: G Alexander (mdalexg@hotmail.com)

\begin{abstract}
Background. Adjuvant lung resection in patients with drug-resistant tuberculosis (DR-TB) not only is cheaper than a 2-month course of drug therapy for multidrug-resistant tuberculosis (MDR-TB) but also, more importantly, has a higher cure rate than medical therapy alone. The cure rate for some MDR-TB patients treated with adjuvant lung resection is about $90 \%$. With the more severe forms of DR-TB, surgical cure rates in selected patients remain high, whereas cure rates decrease when only medical therapy is used. In addition, adjuvant lung resection for DR-TB in selected patients with HIV co-infection does not appear to have a higher complication rate.

Objective. To determine whether specialist pulmonologists in South Africa utilise thoracic surgical intervention for DR-TB appropriately. Methods. A cross-sectional survey was conducted among pulmonologists of the South African Thoracic Society. The study tool was a predesigned, anonymous questionnaire that included 17 closed-ended questions about the role of cardiothoracic surgery in the management of DR-TB.

Results. A 50\% response rate was achieved. The majority of respondents did not know the indications for adjuvant lung surgery in the setting of DR-TB, but would have altered their referral behaviour had they been aware of these indications.

Conclusion. Participating pulmonologists' uncertainty regarding optimal use of adjuvant lung resection for DR-TB suggests the need for local guidelines and education initiatives relevant to the management of these patients. These strategies should include the participation of both the pulmonologist and the cardiothoracic surgeon.
\end{abstract}

Afr J Thoracic Crit Care Med 2018;24(3):107-112. DOI:10.7196/AJTCCM.2018.v24i3.185

Globally, tuberculosis (TB) is the leading cause of death due to a curable infectious disease and was ranked as the leading cause of death in South Africa (SA) already in 2015. ${ }^{[1,2]}$ As a disease of antiquity, it has remained an insurmountable global health challenge and the emergence of drug-resistant tuberculosis (DR-TB) threatens to discount the considerable gains we have made towards disease control and eradication. TB will cost the world's poorest countries up to USD3 trillion over the next 10 years and the World Bank estimates the loss of productivity attributable to TB at $4 \%-7 \%$ of some countries' GDP, as $75 \%$ of TB cases occur in patients between 15 and 54 years of age. ${ }^{[3]}$

In SA, the directly observed treatment short-course (DOTS) strategy achieves a $67 \%$ cure rate while disease recurrence is alarmingly high. ${ }^{[4,5]}$ The combination of disease recurrence, unrestrained disease transmission, limited access to diagnostic services and the absence of highly effective treatment options has fuelled the DR-TB epidemic. ${ }^{[6,7]}$

In SA, medical therapy alone achieves a 6-month culture conversion rate of less than $20 \%$ in HIV-infected patients with extensively drug-resistant tuberculosis (XDR-TB) ${ }^{[8]}$ In 2013, the World Health Organization stated that about $20 \%$ of the 94000 multidrug-resistant tuberculosis (MDR-TB) cases diagnosed in 2012 went untreated. ${ }^{[9,10]}$

Newer drugs such as delamanid, pretomanid and bedaquiline have recently been included in the medical armamentarium, but access to the drugs remains restricted. There is promising evidence that a new standardised regimen for the treatment of MDR-TB may offer the benefits of a shorter treatment duration with improved treatment success. ${ }^{[11]}$ Medical therapy alone for the treatment of MDR-TB has been shown to achieve a cure rate of approximately $54 \%$, while newer medical treatment strategies demonstrate the potential to achieve higher cure rates. ${ }^{[1,12]}$ However, HIV-positive patients were largely excluded from trials of newer drugs and bedaquiline, which was associated with a higher mortality, has been recommended for use only when there is a lack of four effective drugs or when MDR$\mathrm{TB}$ is accompanied by fluoroquinolone resistance. ${ }^{[13,14]}$ Despite the introduction of newer and repurposed drugs, only $80 \%$ of patients with MDR-TB and about $40 \%$ of those with XDR-TB may be cured with appropriate medical therapy alone. ${ }^{[11]}$ Adjuvant lung resection in selected patients has the potential to improve these treatment outcomes further. ${ }^{[8,15-17]}$ The cure rate for selected MDR-TB patients is about $90 \%$ with adjuvant lung resection. ${ }^{[18]}$

In a study by Gandhi et al., ${ }^{[5]} 52$ of 53 XDR-TB patients (98\%) died, with a mean survival of 16 days from the time of specimen collection to death. Of the 44 patients with XDR-TB who consented to testing for HIV, all were found to be co-infected. Although prompt initiation of antiretroviral therapy (ART) in these patients has been demonstrated to improve their outcome, cure rates remain suboptimal. ${ }^{[18]}$ Once again, adjuvant lung resection has an important role in improving the gains of effective medical therapy in this context. ${ }^{[16]}$ 
The emergence of adjuvant lung resection in the management of DRTB will require multidisciplinary teams to identify suitable candidates and to provide pre-, intra- and postoperative care. Such teams might consist of pulmonologists, cardiothoracic surgeons, infectious disease specialists, microbiologists and allied health professionals. As physicians who are entrusted with the medical care of complicated DR-TB patients, pulmonologists will likely be required to initiate a referral to such a multidisciplinary platform. In this survey, we explore the knowledge and practices of SA pulmonologists predominantly with regard to the role of adjuvant lung resection for the management of DR-TB.

\section{Methods}

A cross-sectional survey of all pulmonologists of the South African Thoracic Society was conducted. The South African Thoracic Society is the largest representative professional society of pulmonologists in southern Africa. A list of all registered pulmonologists was made available by the Society and all pulmonologists received an electronic invitation to participate in the survey. The study tool was a predesigned, anonymous questionnaire that included 17 closed-ended questions regarding the role of cardiothoracic surgery in the management of DR-TB. As the study was purely descriptive and no comparisons were made, no further statistical evaluation was performed.

\section{Results}

A response rate of $50 \%$ was achieved, with 25 pulmonologists responding to the survey. The distribution of responses is reported as percentages (Table 1). Approximately three-quarters (76\%) of respondents saw fewer than 10 DR-TB patients per year, while the remaining $24 \%$ saw between 10 and 50 DR-TB patients per year. In addition, $80 \%$ of respondents reported having no access to novel drug therapy for their patients. Almost two-thirds (64\%) of respondents knew the indications for lung resection associated with DR-TB, although a large percentage (60\%) underestimated the potential cure rates for DR-TB with adjuvant lung resection. More than a third (36\%) of the respondents did not recognise the inferior outcomes of medical therapy alone in optimally treated HIV-infected patients compared with HIV-negative patients, and onefifth (20\%) of respondents considered adjuvant lung resection to be associated with poorer outcomes in optimally treated HIV-infected patients than in HIV-negative patients. The majority of respondents $(88 \%)$ indicated that, having been informed of the role of thoracic surgery in the management of DR-TB, they would consider referring their DR-TB patients for surgical assessment in future, despite $92 \%$ never having done so in the past; in this survey, only $8 \%$ of respondents referred every DR-TB patient for a surgical evaluation. Only 32\% of respondents knew what the appropriate timing was for referring patients for surgical assessment after initiating effective drug therapy, while the remainder indicated that such a referral could be made only after at least 6 months. Flexible bronchoscopy, mediastinoscopy, scalene node biopsy and thoracoscopy were the most commonly used invasive thoracic diagnostic modalities used for the diagnosis of DR-TB.

\section{Discussion}

Despite the worsening DR-TB epidemic, there appears to be no effective multidisciplinary management approach in SA. This survey highlights the ineffective utilisation of curative adjuvant lung resection in selected DR-TB patients.
Only $64 \%$ of the respondents were familiar with the indications for adjuvant lung resection associated with DR-TB. Moreover, about half of the respondents were not aware of the cure rate associated with adjuvant lung resection for MDR-TB. It stands to reason that because respondents were unaware of the benefits of adjuvant lung resection, $92 \%$ did not refer their DR-TB patients for a surgical opinion. Unfortunately, no studies have focused on a multidisciplinary approach in treating patients with DR-TB.

There is an alarmingly high mortality with medical therapy alone in patients with DR-TB/HIV co-infection and especially in those with XDR-TB. ${ }^{[5]}$ Although the majority of respondents agreed that adjuvant lung resection is not associated with lower cure rates for DR-TB in HIV-negative patients than in HIV-positive patients who receive ART and who have an undetectable viral load and normal nutrition, this treatment option again appears to have been underutilised. A retrospective study reviewing adjuvant lung resection in patients coinfected with DR-TB/HIV showed cure rates of $75 \%$ and $62 \%$, and conversion rates of $22 \%$ and $38 \%$ in the MDR-TB/HIV-positive group and the XDR-TB/HIV-positive group, respectively. ${ }^{[15]}$ Furthermore, it has been shown that lung resection for massive haemoptysis, whether undertaken electively or emergently and irrespective of a patient's HIV status, does not have a significantly higher complication rate. The CD4 cell count or viral load was considered only in elective cases. ${ }^{[15]}$ In patients who were HIV positive and not on ART, the CD4 cell count had to be $>200$ cells/ $\mu \mathrm{L}$ prior to lung resection; in patients on ART, the viral load had to be undetectable prior to lung resection. ${ }^{[15]}$ Preoperative risk factors such as previous opportunistic lung infections, decreased effort tolerance, a low CD4 cell count without ART, persistently elevated viral load despite ART and nutritional status (in particular, an albumin level $<30 \mathrm{~g} / \mathrm{dL}$ ) may preclude major thoracic surgery. Appropriately selected patients with DR-TB and HIV co-infection may undergo major thoracic surgery with the anticipation of good outcomes. ${ }^{[19]}$

In this survey, $92 \%$ of respondents stated that referral for surgery would be negatively influenced if, after appropriate drug therapy, the patient remains culture positive. It is recommended that patients in whom the isolate is relatively resistant to all antibiotics undergo lung resection within the first 2 months, if feasible. Others should have medical therapy for at least 3 months. After 3 months of medical therapy, the sputum cultures will either be TB-culture negative or have a minimal mycobacterial count. ${ }^{[20]}$ It is essential to undertake surgery before the count rises and it is for these reasons that lung resection is undertaken at this point. An unnecessary delay in surgery may result in the disease spreading beyond the reach of curative surgical resection.

The indications for surgery for DR-TB include: high risk of treatment failure/relapse; 2 or more relapses/ 1 or more relapses while on treatment; persistent sputum positivity after $3-6$ months of therapy within 2 months of surgery; persistent cavitatory disease; intolerance to medical therapy; localised disease equivalent to one lung (absolute criterion); haemoptysis; and bronchiectasis. ${ }^{[20,21]}$

Medical therapy should continue for at least 2 years following surgery. ${ }^{[21]}$ XDR-TB, which occurs in a large proportion of MDR-TB patients, was an independent poor prognostic factor predictor even in HIV-negative patients. ${ }^{[22,23]}$

It has been established that the bacillary load content of pulmonary TB cavities is $10^{7}-10^{9}$ and that of pulmonary TB nodules is $10^{2}-$ 


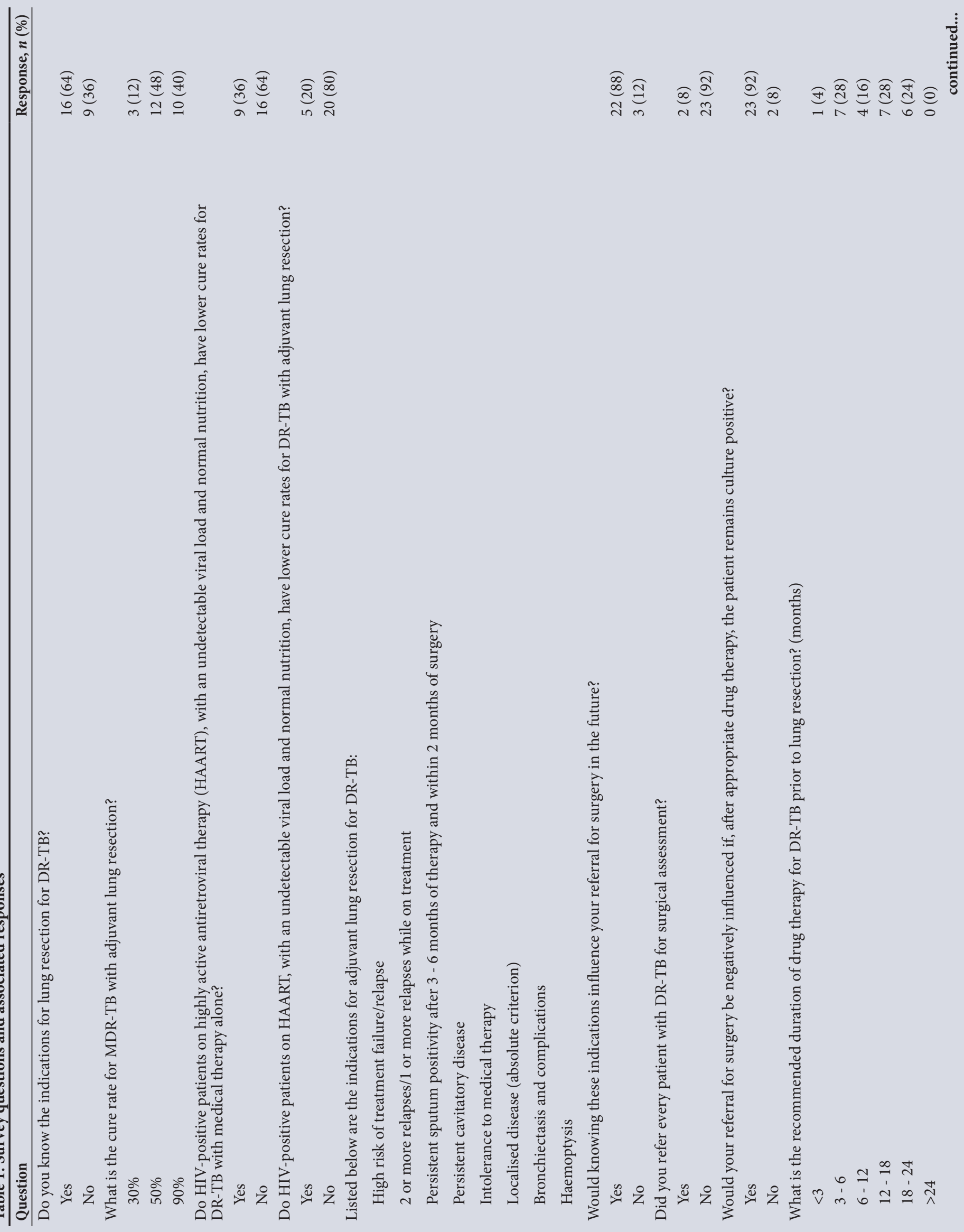




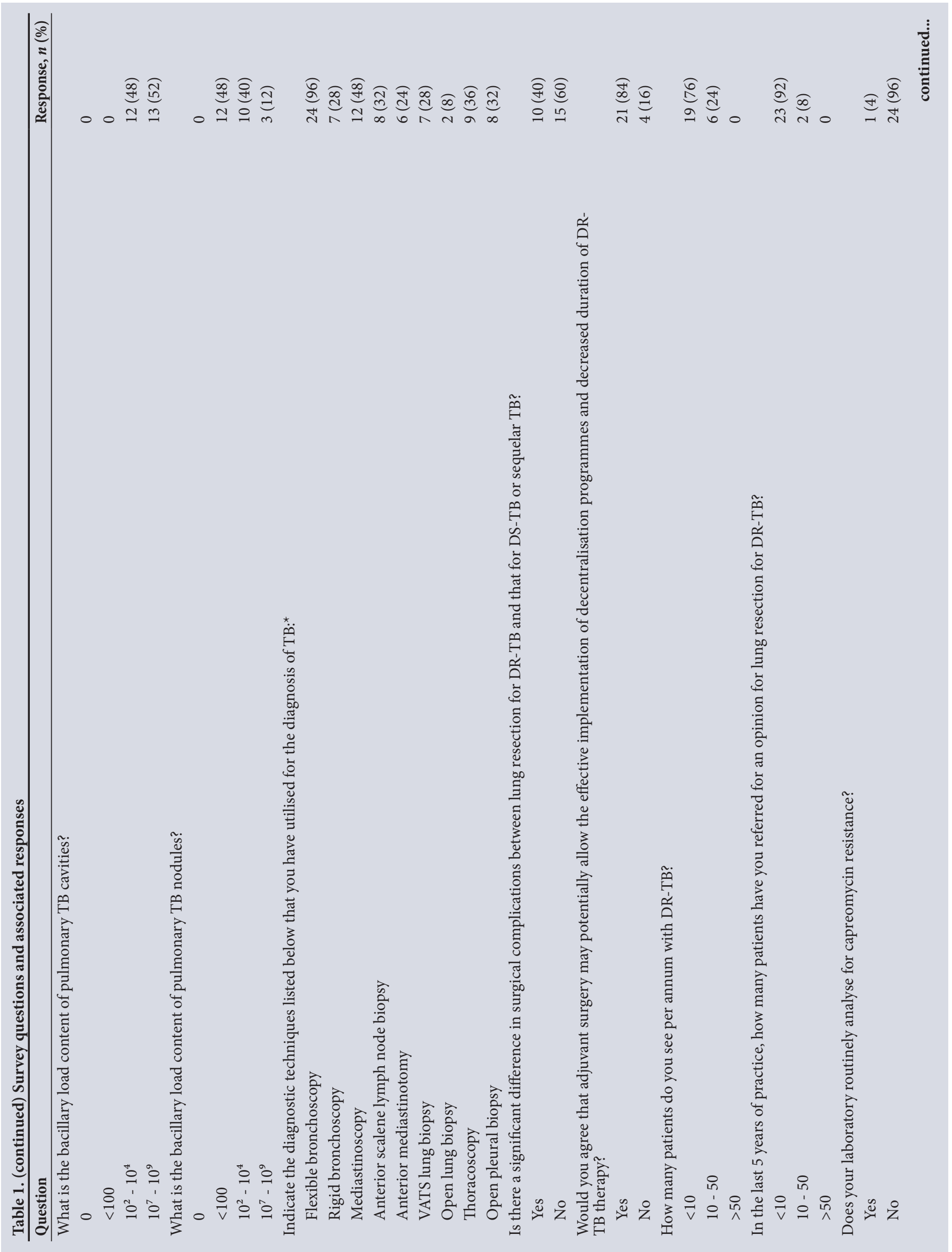




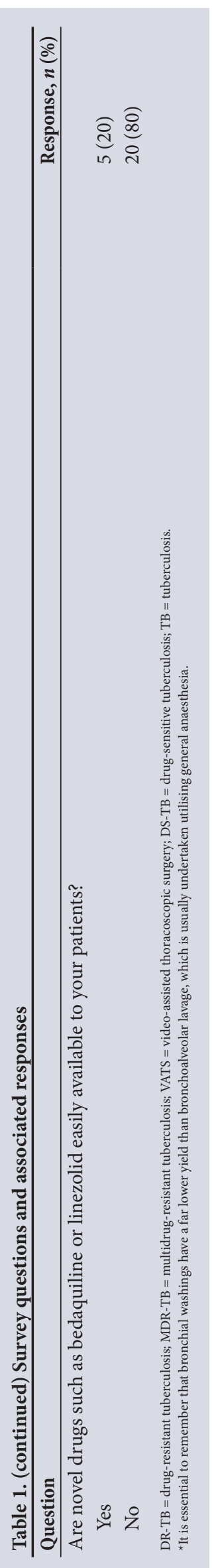

$10^{4}$ bacilli, yet only $52 \%$ and $40 \%$ of respondents, respectively, correctly acknowledged these facts. ${ }^{[20]}$ As such, patients with lung cavities and nodules are at a high risk of recurrence, even if sputum-negative. Moreover, drug penetration of the cavities and nodules is suboptimal.

Despite excellent results with adjuvant lung resection, albeit in selected patients, medical therapy still remains a virtually exclusive form of management among most of the physicians who participated in this survey. This appears to be the exclusive result of a lack of knowledge regarding the benefits of and indications for adjuvant lung resection. We suggest that a national multidisciplinary team be established to formalise treatment guidelines for adjuvant lung resection in DR-TB patients, irrespective of HIV status. This should include patient selection and timing of surgery based on current practices in the various cardiothoracic units in SA. Surgical outcomes in the various units making proposals should be taken into account.

It is the authors' opinion that such guidelines should be formally endorsed by the Department of Health, once established. All units treating DR-TB patients should comprise a mandatory multidisciplinary team. Every opportunity should be used to ensure that these guidelines are accessible to the relevant healthcare practitioners, for example through TB awareness road shows, media campaigns, journal publications and local congresses.

A national DR-TB register to collect relevant surgical data is also recommended. It should include standardised radiological reports, reasons for proposing or declining adjuvant lung resection and a record of surgical outcomes. Such a registry would allow participation from both the public and the private sectors.

\section{Study limitations}

The small number of respondents is an obvious limitation and the results of this study may not be generalisable to other settings. Furthermore, the small sample size precluded further statistical evaluation and the identification of potentially useful predictors of practitioner perceptions or behaviour.

\section{Conclusion}

DR-TB is, and will continue to be, a formidable adversary, which requires role players to deal collectively, effectively and timeously with patients presenting with this disease. The uncertainty regarding optimal utilisation of adjuvant lung resection for DRTB patients among pulmonologists suggests the need for educational strategies relevant to the optimal management of these patients. These strategies should include the participation of both the pulmonologist and the cardiothoracic surgeon. This survey further highlights the lack of a multidisciplinary approach to effectively implement appropriate management of DR-TB.

Acknowledgements. None.

Author contributions. GA: compiled and distributed survey, collected and analysed data and wrote the manuscript; RP: assessed statistics and edited the manuscript.

Funding. None.

Conflicts of interest. None.

1. World Health Organization (WHO). Global Tuberculosis Report 2015. Geneva: WHO, 2015.

2. Statistics South Africa (Stats SA). Mortality and causes of death in South Africa, 2015: Findings from death notification. Pretoria: Stats SA, 2017.

3. TB Alliance. The Cycle of Poverty. https://www.tballiance.org/whynew-tb-drugs/cycle-poverty (accessed 30 August 2018).

4. Pooran A, Pieterson E, Davids M, Theron G, Dheda K. What is the cost of diagnosis and management of drug resistant tuberculosis in South Africa? PLoS ONE 2013;8(1):e54587. https://doi.org/10.1371/ journal.pone.0054587

5. Gandhi NR, Moll A, Sturm AW, et al. Extensively drug-resistant tuberculosis as a cause of death in patients co-infected with tuberculosis and HIV in a rural area of South Africa. Lancet 2006;368:1575-1580. https://doi.org/10.1016/s0140-6736(06)69573-1

6. National Department of Health (NDoH). Management of Drugresistant Tuberculosis: Policy Guidelines. Pretoria: NDoH, 2013.

7. National Institute for Communicable Diseases (NICD). South African Tuberculosis Drug Resistance Survey 2012-14. Johannesburg: NICD, 2016.

8. Calligaro GL, Moodley L, Symons G, Dheda K. The medical and surgical treatment of drug-resistant tuberculosis. J Thorac Dis 2014;6(3):186-195. https://doi.org/10.3978/j.issn.20721439.2013.11.11

9. World Health Organization (WHO). Global tuberculosis control: WHO Report 2011. Geneva: WHO, 2011.

10. Marks SM, Flood J, Seaworth B, et al. Treatment practices, outcomes and costs of multidrug-resistant and extensively drugresistant tuberculosis, United States, 2005-2007. Emerg Infect Dis 2014;20(5):812-820. https://doi.org/10.3201/eid2005.131037

11. Moodley R, Godec TR, STREAM Trial Team. Short-course treatment for multidrug-resistant tuberculosis: The STREAM trials. Eur Respir Rev 2016;25(139):29-35. https://doi.org/10.1183/16000617.00802015

12. Ahuja SD, Ashkin D, Avendano M, et al. Multidrug resistant pulmonary tuberculosis treatment regimens and patient outcomes: An individual patient data meta-analysis of 9,153 patients. PLoS Med 2012;9(8):e1001300. https://doi.org/10.1371/journal.pmed.1001300

13. Gler MT, Skripconoka V, Sanchez-Garavito E, et al. Delamanid for multidrug-resistant pulmonary tuberculosis. N Engl J Med 2012;366(23):2151-2160. https://doi.org/10.1056/NEJMoa1112433

14. Diacon AH, Pym A, Grobusch MP, et al. Multidrug-resistant tuberculosis and culture conversion with bedaquiline. $\mathrm{N} \mathrm{Engl} \mathrm{J} \mathrm{Med}$ 2014;371(8):723-732. https://doi.org/10.1056/NEJMc1412235

15. Alexander GR, Biccard B. A retrospective review comparing treatment outcomes of adjuvant lung resection for drug-resistant tuberculosis in patients with and without human immunodeficiency virus co-infection. Eur J Cardiothorac Surg 2016;49(3):823-828. https://doi.org/10.1093/ejcts/ezv228

16. Maharaj K, Perumal R, Alexander G. Pulmonary nodules in patients with drug-resistant tuberculosis who have undergone adjuvant lung resection in a high-HIV-burden setting. S Afr Respir J 2017;23(3):5560. http://dx.doi.org/10.7196/SARJ.2017.v23i3.174

17. Lalloo UG, Naidoo R, Ambaram A. Recent advances in the medical and surgical treatment of multi-drug resistant tuberculosis. Curr Opin Pulm Med 2006;12(3):179-185. https://doi.org/10.1097/01. mcp.0000219266.27439.52 
18. O’Donnell MR, Padayatchi N, Master I, Osburn G, Horsburgh CR. Improved early results for patients with extensively drug-resistant tuberculosis and HIV in South Africa. Int J Tuberc Lung Dis 2009;13(7):855-861.

19. Filsoufi F, Salzberg SP, Von Harbou KTJ, Neibart E, Adams DH. Excellent outcomes of cardiac surgery in patients infected with HIV in the current era. Clin Infect Dis 2006;43(4):532-536. https://doi.org/10.1086/505977

20. Pomerantz BJ, Cleveland JC, Olson HK, Pomerantz M. Pulmonary resection for multidrug resistant tuberculosis. J Thorac Cardiovasc Surg 2001;121(3):448-453. https:// doi.org/10.1067/mtc.2001.112339

21. Furák J, Troján I, Szöke T, et al. Surgical intervention for pulmonary tuberculosis: Analysis of indications and perioperative data relating to diagnostic and therapeutic resections. Eur J Cardiothorac Surg 2001;20:722-727. https://doi.org/10.1016/s1010 7940(01)00874-0
22. Shiraishi Y, Katsuragi N, Kita H, Toishi M, Onda T. Experience with pulmonary resection for extensively drug-resistant tuberculosis. Interact Cardiovasc Thorac Surg 2008;7:1075-1078. https://doi.org/10.1510/icvts.2008.185124

23. Kim DH, Kim HJ, Park SK, et al. Treatment outcomes and long-term survival in patients with extensively drug-resistant tuberculosis. Am J Respir Crit Care Med 2008;178(10):1075-1082. https://doi.org/10.1164/rccm.200801-132OC

Accepted 26 February 2018 